\title{
NAVIER-STOKES APPROXIMATIONS TO 2D VORTEX SHEETS IN HALF PLANE*
}

\author{
DONGJUAN NIU ${ }^{\dagger}$, QUANSEN JIU ${ }^{\ddagger}$, AND ZHOUPING XIN§
}

\begin{abstract}
This paper concerns the two-dimensional Euler equations with vortex-sheets initial data in half plane and in the domain $\Omega=\left\{\left(x_{1}, x_{2}\right): x_{2} \geq \gamma\left(x_{1}\right)\right\}, \gamma\left(x_{1}\right)=0$ for $\left|x_{1}\right| \geq x_{0}, x_{0}$ is a fixed constant, and $\gamma\left(x_{1}\right)$ is a sufficient smooth and simple curve. The Navier-Stokes approximations are constructed in this paper and by means of vanishing the viscosity, the global existence of weak solutions is obtained under the assumption that the initial vorticity is of one-sign. Navier boundary conditions are applied when constructing the Navier-Stokes approximations.
\end{abstract}

Key words. Vanishing viscosity limit, Euler equations, vortex-sheets data, Navier-Stokes approximations.

AMS subject classifications. 76B47, 35Q30

1. Introduction. We consider the following two-dimensional incompressible Euler equations

$$
\left\{\begin{aligned}
\partial_{t} u+u \cdot \nabla u+\nabla p=0 & & x \in \mathbb{H}, t>0 \\
\operatorname{div} u=0 & & x \in \mathbb{H}, t>0 \\
|u(x, t)| \rightarrow 0 & & |x| \rightarrow \infty
\end{aligned}\right.
$$

where $\mathbb{H}=\left\{\left(x_{1}, x_{2}\right): x_{2} \geq 0\right\}$. Define $\Gamma \doteq \partial \mathbb{H}=\left\{x_{2}=0\right\}$. The unknown functions $p=p(x, t)$ and $u=\left(u_{1}(x, t), u_{2}(x, t)\right)$ represent the pressure and velocity fields function, respectively.

The initial and boundary conditions of (1.1) are imposed as

$$
u(x, t=0)=u_{0} \quad x \in \mathbb{H},
$$

and

$$
u \cdot n=0 \text { on } \Gamma \text {. }
$$

In (1.3), $n$ means the unit normal vector of $\Gamma$.

The vorticity of the velocity $u(x, t)$ is denoted by $\omega(x, t)=\operatorname{curl} u$ and the initial vorticity is given by $\omega_{0}(x)=$ curl $u_{0}$. Roughly speaking, for a general domain $\Omega \subseteq \mathbb{R}^{2}$, the initial data of (1.1) are called vortex-sheets data if the initial velocity is locally square integrable and the initial vorticity is a finite Radon measure,that is, $u_{0} \in$

\footnotetext{
*Received February 1, 2008; accepted for publication April 21, 2008.

${ }^{\dagger}$ School of Mathematical Sciences, Capital Normal University, Beijing 100048, P. R. China ( niuniudj@gmail.com). The author is partially supported by National Natural Sciences Foundation of China (No.10871133).

${ }_{\ddagger}^{\ddagger}$ School of Mathematical Sciences, Capital Normal University, Beijing 100048, P. R. China (jiuqs@ mail.cnu.edu.cn). The author is supported partially by National Natural Sciences Foundation of China (No.10871133 \& No. 10771177), Zheng Ge Ru Founds and Grants from RGC of HKSAR CUHK4028/04P, CUHK4040/06P and RGC Central Allocation Grant CA05/06.SC01.

$\S$ The Institute of Mathematical Sciences, The Chinese University of Hong Kong, Shatin, N.T., Hong Kong (zpxin@ims.cuhk.edu.hk). The author is supported by Zheng Ge Ru Founds, Grants from RGC of HKSAR CUHK4028/04P, CUHK4040/06P and RGC Central Allocation Grant CA05/06.SC01.
} 
$L_{\text {loc }}^{2}(\Omega), \omega_{0} \in B M(\Omega)$, where $B M(\Omega)$ is the finite Radon measure spaces. There are a lot of studies in recent years on the existence of weak solutions of (1.1) when the initial data are vortex-sheets data. In 1991, Delort first proved the global existence of weak solutions to (1.1) under the assumption that the initial vorticity is a vortexsheets data and of one-sign (see [1]). The approximate solutions were constructed through mollifying the initial data in [1]. Later on, for one-sign initial vorticity, the convergence to the classical weak solutions of vortex-sheets problem for various types of the approximate solutions has been established (see [4], [16], [17]). In particular, for the Cauchy problem, the Navier-Stokes approximations were constructed by Majda in [16] and the vortex-method approximations were constructed by Liu and Xin in [11] and [12]. In the case that the vorticity changes signs, Lopes Filho, Nussenzveig Lopes and Xin established in [14] the global existence of weak solutions to the twodimensional vortex-sheets problem under the assumption that initial vorticity holds reflection symmetry. In [15], the authors generalized the above result to the case of exterior domain which is symmetric with respect to the $x_{1}$ axis. The existence of weak solutions of two-dimensional incompressible Euler equations with $\omega_{0} \in L^{1}\left(\mathbb{R}^{2}\right)$ was studied in [19]. Some studies on the convergence of the approximate solutions of the three-dimensional axisymmetric Euler equations are referred to [2] and [7]-[9].

It should be noted that in [14] the authors constructed the approximate solutions by smoothing the initial data. And in [14] the authors pointed out a question whether the approximate solutions of (1.1) can be constructed by the solutions of the Navier-Stokes equations with no-slip boundary condition $u=0$ on $\Gamma$. In this paper, we partially answer the question through constructing the Navier-Stokes approximations by using the free Navier boundary conditions (see (1.5)) instead of the no-slip boundary conditions. More precisely, we consider the following Navier-Stokes system

$$
\left\{\begin{aligned}
\partial_{t} u^{\epsilon}+u^{\epsilon} \cdot \nabla u^{\epsilon}+\nabla p^{\epsilon}-\epsilon \Delta u^{\epsilon} & =0 & & x \in \mathbb{H}, t>0, \\
\operatorname{div} u^{\epsilon} & =0 & & x \in \mathbb{H}, t>0, \\
\left|u^{\epsilon}\right|(x, t) & \rightarrow 0 & & \text { as }|x| \rightarrow \infty,
\end{aligned}\right.
$$

with the free Navier boundary conditions as

$$
\omega^{\epsilon}(x, t)=\operatorname{curl} u^{\epsilon}=0, u^{\epsilon} \cdot n=0 \quad x \in \Gamma, t>0,
$$

and initial data as

$$
u^{\epsilon}(x, t=0)=u_{0}^{\epsilon}, \quad \omega^{\epsilon}(x, t=0)=\omega_{0}^{\epsilon} \quad x \in \mathbb{H} .
$$

In (1.6), the initial vorticity $\omega_{0}^{\epsilon}$ is a smooth and compactly supported function such that $\omega_{0}^{\epsilon}$ tends to $\omega_{0}$ as $\epsilon \rightarrow 0$ in $B M(\mathbb{H})$. Then by the classical theory of the $2 \mathrm{D}$ incompressible Navier-Stokes equations (see [10] for instance), there exists a unique smooth solution $\omega^{\epsilon}$ to the initial-boundary problem (1.4)-(1.6). Our main task of this paper is to take the limit of $\omega^{\epsilon}$ as $\epsilon$ tends to zero and obtain that the limit $\omega$ is the weak solution of $2 \mathrm{D}$ Euler equations when the initial data are vortex-sheets ones. A new ingredient of this paper is that we use the Navier-Stokes approximations and the Navier boundary conditions (1.5) are applied. In fact, we can generalize our results to the domain $\Omega=\left\{\left(x_{1}, x_{2}\right): x_{2} \geq \gamma\left(x_{1}\right)\right\}$, where $\gamma$ is a simple and smooth curve, and even more generally, to the exterior domain with reflection symmetry.

Before end this section, we point out that the general Navier boundary conditions, which were first used by Navier in 1827, mean that there is a stagnant layer of fluid 
close to the wall allowing a fluid to slip, and the slip velocity is proportional to the shear stress (see [5], [6],[13],[20] and references therein). The Navier boundary condition (1.5), which is called a free Navier boundary condition, is a special form of the general Navier boundary conditions (see [20]).

The rest of the paper is organized as follows. In Section 2, we present some preliminaries and the definition of weak solutions of (1.1). In Section 3, by a priori estimates, it is obtained that the convergence of viscous approximates to a weak solution of (1.1)-(1.3) in $\mathbb{H}$. In Section 4, the cases of more general domain are discussed.

2. Some preliminaries. By Biot-Savart law, the velocity can be recovered from the vorticity in the following way.

$$
u=u(x, t)=K_{\mathbb{H}}[\omega](x, t) \equiv \int_{\mathbb{H}} K_{\mathbb{H}}(x, y) \omega(y, t) d y,
$$

where

$$
K_{\mathbb{H}}(x, y)=\frac{(x-y)^{\perp}}{2 \pi|x-y|^{2}}-\frac{\left(x-y^{*}\right)^{\perp}}{2 \pi\left|x-y^{*}\right|^{2}}
$$

with $x^{\perp}=\left(-x_{2}, x_{1}\right)$ and $x^{*}=\left(x_{1},-x_{2}\right)$. Let $\omega_{0}=\omega_{0}(x) \in B M(\mathbb{H})$ be a Radon measure with compact support such that $u_{0}=K_{\mathbb{H}}\left[\omega_{0}\right] \in\left(L^{2}(\mathbb{H})\right)^{2}$.

From (1.4)-(1.6), the initial-boundary problem of the vorticity equation of the 2D Navier-Stokes equations can be expressed as

$$
\left\{\begin{array}{rl}
\omega_{t}^{\epsilon}+u^{\epsilon} \cdot \nabla \omega^{\epsilon}=\epsilon \Delta \omega^{\epsilon} & x \in \mathbb{H}, t>0, \\
\operatorname{div} u^{\epsilon}=0, \operatorname{curl} u^{\epsilon}=\omega^{\epsilon} & x \in \mathbb{H}, t>0 \\
u^{\epsilon} \cdot n=0, \omega^{\epsilon}=0 & x \in \Gamma, t>0 \\
\omega^{\epsilon}(x, t=0)=\omega_{0}^{\epsilon}(x) & x \in \mathbb{H}, \\
\left|u^{\epsilon}(x, t)\right| \rightarrow 0 & \text { as }|x| \rightarrow \infty .
\end{array}\right.
$$

Define the set of the admissible test functions by

$$
\mathcal{A} \equiv\left\{\varphi \in C^{\infty}([0, \infty) \times \overline{\mathbb{H}}) \mid \varphi \equiv 0 \text { on } \Gamma\right\} .
$$

Then the definition of the weak solutions of (1.1)-(1.3) can be defined as (see [14])

Definition 2.1. The function $\omega \in L^{\infty}\left([0, \infty) ; B M_{l o c}(\mathbb{H})\right)$ is called a weak solution of the incompressible 2D Euler equations with initial data $\omega_{0}$ if:

(i) the velocity $u \equiv K_{\mathbb{H}}[\omega]$ belongs to $L_{l o c}^{\infty}\left([0, \infty) ; L^{2}(\mathbb{H})^{2}\right)$, and

(ii) for any test function $\varphi \in \mathcal{A}$, it holds that

$$
\begin{aligned}
& \int_{0}^{\infty} \int_{\mathbb{H}} \varphi_{t} \omega(x, t) d x d t+\int_{\mathbb{H}} \varphi(x, 0) \omega_{0}(x) d x \\
& +\int_{0}^{\infty} \int_{\mathbb{H}} \int_{\mathbb{H}} H_{\varphi}(x, y, t) \omega(x, t) \omega(y, t) d y d x d t=0,
\end{aligned}
$$

where $H_{\varphi}(x, y, t) \equiv \frac{1}{2}\left(\nabla \varphi(x, t) \cdot K_{\mathbb{H}}(x, y)+\nabla \varphi(y, t) \cdot K_{\mathbb{H}}(y, x)\right)$.

REMARK 2.1. The weak solution defined above is called a "boundary-coupled weak solution" in [14], and it is stronger than the standard one in the sense that we only require that the test function vanishes at the boundary.

REMARK 2.2. $H_{\varphi}(x, y, t)$ is globally bounded in $\mathbb{H} \times \mathbb{H} \times[0, \infty)($ see $[17])$. 
3. Convergence theorem. We will begin with a reasonably straightforward generalization of the argument used in [14] to show that the vorticity has no concentration up to the boundary. This argument mainly consists of the following two lemmas.

Lemma 3.1. Let $\omega^{\epsilon}=\omega^{\epsilon}(x, t)$ be a nonnegative and smooth solution of (2.2). Let $\varphi=\varphi(x)$ be a nonnegative smooth function on $\mathbb{H}$ with bounded derivatives up to second order. Then the following inequality holds:

$$
\begin{aligned}
& \frac{d}{d t} \int_{\mathbb{H}} \varphi(x) \omega^{\epsilon}(x, t) d x \\
& \leq \int_{\Gamma}\left(\frac{1}{2}\left|u^{\epsilon} \cdot n^{\perp}\right|^{2} \nabla \varphi \cdot n^{\perp}\right) d S+\epsilon \int_{\mathbb{H}} \Delta \varphi \omega^{\epsilon} d x \\
& \quad+\int_{\mathbb{H}}\left[\left(\left(u_{1}^{\epsilon}\right)^{2}-\left(u_{2}^{\epsilon}\right)^{2}\right) \varphi_{x_{1} x_{2}}-u_{1}^{\epsilon} u_{2}^{\epsilon}\left(\varphi_{x_{1} x_{1}}-\varphi_{x_{2} x_{2}}\right)\right] d x
\end{aligned}
$$

Proof. For simplicity, $\left(u^{\epsilon}, \omega^{\epsilon}\right)$ will be denoted by $(u, \omega)$ in the proof. Using the equations in system (2.2), it holds that

$$
\begin{aligned}
\frac{d}{d t} \int_{\mathbb{H}} \varphi(x) \omega(x, t) d x & =\int_{\mathbb{H}} \varphi(x) \omega_{t} d x \\
& =-\int_{\mathbb{H}} \operatorname{div}(u \omega) \varphi d x+\epsilon \int_{\mathbb{H}} \varphi \Delta \omega d x \\
& =\int_{\mathbb{H}}(u \cdot \nabla \varphi) \omega d x+\epsilon \int_{\Gamma} \frac{\partial \omega}{\partial n} \varphi d S+\epsilon \int_{\mathbb{H}} \Delta \varphi \omega d x \\
& \equiv I_{1}+I_{2}+I_{3} .
\end{aligned}
$$

Since $\omega=-\operatorname{div} u^{\perp}$, then

$$
\begin{aligned}
I_{1} & =\int_{\mathbb{H}}(u \cdot \nabla \varphi) \cdot\left(-\operatorname{div} u^{\perp}\right) d x \\
& =\int_{\mathbb{H}} \nabla(u \cdot \nabla \varphi) \cdot u^{\perp} d x-\int_{\Gamma}(u \cdot \nabla \varphi) \cdot\left(u^{\perp} \cdot n\right) d S .
\end{aligned}
$$

Note that

$$
\nabla(\nabla \varphi \cdot u) \cdot u^{\perp}=\nabla\left(\frac{|u|^{2}}{2}\right) \cdot \nabla^{\perp} \varphi+\left(u_{1}^{2}-u_{2}^{2}\right) \varphi_{x_{1} x_{2}}-u_{1} u_{2}\left(\varphi_{x_{1} x_{1}}-\varphi_{x_{2} x_{2}}\right) .
$$

Therefore,

$$
\begin{aligned}
I_{1}= & \int_{\mathbb{H}}\left[\nabla\left(\frac{|u|^{2}}{2}\right) \cdot \nabla^{\perp} \varphi+\left(u_{1}^{2}-u_{2}^{2}\right) \varphi_{x_{1} x_{2}}\right. \\
& \left.-u_{1} u_{2}\left(\varphi_{x_{1} x_{1}}-\varphi_{x_{2} x_{2}}\right)\right] d x-\int_{\Gamma}(u \cdot \nabla \phi)\left(u^{\perp} \cdot n\right) d S \\
= & \int_{\mathbb{H}}\left[\left(u_{1}^{2}-u_{2}^{2}\right) \varphi_{x_{1} x_{2}}-u_{1} u_{2}\left(\varphi_{x_{1} x_{1}}-\varphi_{x_{2} x_{2}}\right)\right] d x \\
& +\int_{\Gamma}\left(\frac{|u|^{2}}{2} \nabla^{\perp} \varphi-(\nabla \varphi \cdot u) u^{\perp}\right) \cdot n d S,
\end{aligned}
$$


where we used the decay of $|u|$ at infinity. Due to the boundary condition $\omega=0, x \in \Gamma$, and the nonnegativity of $\omega^{\epsilon}$ and $\varphi$, one has

$$
I_{2}=-\epsilon \int_{\Gamma} \frac{\partial \omega}{\partial x_{2}} \varphi d S \leq 0
$$

In addition, by direct calculation, one has

$$
\int_{\Gamma}\left(\frac{|u|^{2}}{2} \nabla^{\perp} \varphi-(\nabla \varphi \cdot u) u^{\perp}\right) \cdot n d S=\int_{\Gamma} \frac{1}{2}\left|u \cdot n^{\perp}\right|^{2} \nabla \varphi \cdot n^{\perp} d S .
$$

where the boundary condition $u \cdot n=0$ on $\left\{x_{2}=0\right\}$ has been used. Putting (3.4) and the above equality into (3.2), we obtain (3.1) and the proof of the lemma is complete.

Remark 3.1. Actually, in (3.1), one has

$$
\int_{\Gamma}\left(\frac{1}{2}\left|u^{\epsilon} \cdot n^{\perp}\right|^{2} \nabla \varphi \cdot n^{\perp}\right) d S=\frac{1}{2} \int_{-\infty}^{+\infty}\left(u_{1}^{\epsilon}\left(x_{1}, 0, t\right)\right)^{2} \varphi_{x_{1}}\left(x_{1}, 0\right) d x_{1} .
$$

Choosing $\phi=\operatorname{arcctg}\left(x_{1}\right), x_{1} \in[-L, L]$ in (3.1), integrating in time, we have the following a priori estimate on $u_{1}\left(x_{1}, 0, t\right)$

$$
\int_{0}^{T} \int_{-L}^{L}\left|u_{1}^{\epsilon}\left(x_{1}, 0, t\right)\right|^{2} d x_{1} d t \leq C
$$

where $L$ is an any constant satisfying $0<L<\infty$, and $C$ is a constant depending on $\left\|\omega_{0}\right\|_{L^{1}},\left\|u_{0}\right\|_{L^{2}}, T$ and $L$. This can be proved in a completely similar way presented in [14] and we omit it here.

It follows from (3.5) that the total mass of the vorticity in a disk around a point on the interface $\left\{x_{2}=0\right\}$ decays as the disk shrinks to a point. More precisely,

Lemma 3.2. Let $\left(u^{\epsilon}, \omega^{\epsilon}\right)$ be the smooth solution of (1.4) as in Lemma 3.1. Set $x^{0}=(a, 0) \in \mathbb{R}^{2}$. If $L>0$ and $\delta>0$ are such that $(a-\delta, a+\delta) \subset(-L, L)$, then

$$
\int_{B\left(x^{0}, \delta\right) \cap \mathbb{H}} \omega^{\epsilon}(y, t) d y \leq C \sqrt{\delta}\left(\int_{-L}^{L}\left|u_{1}^{\epsilon}\left(x_{1}, 0, t\right)\right|^{2} d x_{1}\right)^{\frac{1}{2}},
$$

where $C$ is a universal constant.

The proof of Lemma 3.2 is referred to that of Lemma 2 in [14].

The key issue in the proof of the global existence of weak solutions, as formulated by Schochet in [17], is to control the possible concentrations in the limit process of the sequence $\left\{\omega^{\epsilon}\right\}$ as $\epsilon \rightarrow 0$. To this end, we will use a priori estimate (3.5) and the $\log ^{-\frac{1}{2}}$ decay of circulation in small circles, first observed by Majda in [16]. In fact, the a priori logarithmic decay in circulation turns out to be a local feature of flows with one-sign vorticity, which was pointed out by Schochet in [17]. More precisely, we have

THEOREM 3.3. Under the assumptions of Lemma 3.2, for every $T>0$, and each compact set $\Sigma \subseteq \overline{\mathbb{H}}$, there exists a constant $C>0$ such that for any $0<\delta<1$,

$$
\int_{0}^{T}\left(\sup _{x \in \Sigma} \int_{B(x, \delta) \cap \mathbb{H}} \omega^{\epsilon}(y, t) d y\right) d t \leq C|\log \delta|^{-\frac{1}{2}} .
$$


Proof. This proof can be seen in [14]. For completeness, we just list the sketch of the proof here. Fix $\Sigma \subseteq B(0, R) \cap \mathbb{H}$ with $R>0$ and $0<\delta<1$. Define a cut off function as

$$
\eta_{\delta}(z)=\left\{\begin{array}{l}
1 \quad \text { if } \quad|z| \leq \delta \\
\frac{\log (|z|)-\log (\sqrt{\delta})}{\log (\sqrt{\delta})} \text { if } \delta \leq|z| \leq \sqrt{\delta} \\
0 \quad \text { if }|z| \geq \sqrt{\delta} .
\end{array}\right.
$$

Integrating by parts and using the fact that $\omega^{\epsilon}=\nabla^{\perp} \cdot u^{\epsilon}$, one has

$$
\begin{aligned}
\int_{B(x, \delta) \cap \mathbb{H}} \omega^{\epsilon}(y, t) d y & \leq \int_{B(x, \sqrt{\delta}) \cap \mathbb{H}} \eta_{\delta}(x-y) \omega^{\epsilon}(y, t) d y \\
& =\int_{B(x, \sqrt{\delta}) \cap \mathbb{H}} \nabla_{y} \eta_{\delta}(x-y) \cdot\left(u^{\epsilon}\right)^{\perp}(y, t) d y \\
& +\int_{B(x, \sqrt{\delta}) \cap \Gamma} \eta_{\delta}(x-y) \cdot u^{\epsilon}(y, t) \cdot n^{\perp} d S .
\end{aligned}
$$

Note that the other boundary terms vanish since $\eta_{\delta}(x-y)=0$ for $y \in \partial B(x, \sqrt{\delta})$. Therefore,

$$
\begin{aligned}
& \int_{B(x, \delta) \cap \mathbb{H}} \omega^{\epsilon}(y, t) d y \\
& \leq C|\log \delta|^{-\frac{1}{2}}\left\|u_{0}\right\|_{L^{2}(\mathbb{H})} \\
& \quad+\left(\int_{B(x, \sqrt{\delta}) \cap \Gamma}\left|u^{\epsilon} \cdot n^{\perp}\right|^{2} d S\right)^{\frac{1}{2}}|B(x, \sqrt{\delta}) \cap \Gamma|^{\frac{1}{2}}
\end{aligned}
$$

Finally, it follows easily that $|B(x, \sqrt{\delta}) \cap \Gamma| \leq C \sqrt{\delta}$, which, together with (3.5), yields the desired estimate. The proof of the theorem is complete.

Now we are ready to obtain the existence to the problem (1.1)-(1.3) by using the Navier-Stokes approximations (1.4)-(1.5).

THEOREM 3.4. Suppose that $\omega_{0} \in B M(\mathbb{H})$ is nonnegative and has a compact support such that $u_{0}=K_{\mathbb{H}}\left[\omega_{0}\right] \in\left(L^{2}(\mathbb{H})\right)^{2}$. Then the weak limit $w \in L^{\infty}(0, \infty ; B M(\mathbb{H}))$ of the Navier-Stokes approximations (1.4)-(1.5) is a weak solution of (1.1)-(1.3).

Proof. Let $\zeta=\zeta(x) \in C_{c}^{\infty}([0, \infty))$ be nonnegative and monotonically decreasing inside its support, with total integral 1, and fix the mollifier $\psi(x)=\zeta(|x|)$. Consider a sequence of smooth and compactly supported functions $\left\{\omega_{0}^{\epsilon}\right\}$, obtained by convolving $\omega_{0}$ with $\psi^{\epsilon}=\psi^{\epsilon}(x)=\frac{1}{\epsilon^{2}} \psi\left(\frac{x}{\epsilon}\right)$. As shown in [3], $\omega_{0}^{\epsilon}$ is uniformly bounded in $L^{1}(\mathbb{H})$ and $u_{0}^{\epsilon}=K_{\mathbb{H}}\left[\omega_{0}\right] \in\left(L^{2}(\mathbb{H})\right)^{2}$. Due to the classical theory of the incompressible NavierStokes equations (see [10], [18]), there exists a smooth approximate solution $\left(u^{\epsilon}, \omega^{\epsilon}\right)$ of $(2.2)$.

In addition, as shown in [3], the following estimate holds for all $T>0$,

$$
\sup _{0 \leq t \leq T}\left\|\omega^{\epsilon}\right\|_{L^{1}(\mathbb{H})} \leq C
$$

where the fact that $\epsilon \int_{\Gamma} \frac{\partial \omega^{\epsilon}}{\partial n} \leq 0$ has been used. Moreover, it holds that 


$$
\begin{aligned}
\frac{1}{2} \frac{d}{d t}\left\|u^{\epsilon}\right\|_{L^{2}(\mathbb{H})}^{2}+\left\|\nabla u^{\epsilon}\right\|_{L^{2}(\mathbb{H})}^{2} & \leq C\left|u^{\epsilon}\right|_{L^{2}(\Gamma)}^{2} \leq C\left\|u^{\epsilon}\right\|_{L^{2}(\mathbb{H})}\left\|\nabla u^{\epsilon}\right\|_{L^{2}(\mathbb{H})} \\
& \leq C\left\|u^{\epsilon}\right\|_{L^{2}(\mathbb{H})}+\frac{1}{2}\left\|\nabla u^{\epsilon}\right\|_{L^{2}(\mathbb{H})}^{2},
\end{aligned}
$$

where the identity $\int_{\Gamma} \frac{\partial u^{\epsilon}}{\partial n} \cdot u^{\epsilon}=\frac{1}{2} \int_{\Gamma}\left|u^{\epsilon}\right|^{2}$ is used, due to the free boundary condition (1.5) (see [10]). Therefore, we have

$$
\sup _{0 \leq t \leq T}\left\|u^{\epsilon}\right\|_{L^{2}(\mathbb{H})} \leq C .
$$

Moreover, it is easy to obtain that there exists a $1<M<\infty$, such that $\left\{\omega_{t}^{\epsilon}\right\}$ is uniformly bounded in $\operatorname{Lip}\left([0, T] ; H_{l o c}^{-M}(\mathbb{H})\right)$. In fact, letting $\varphi \in C_{c}^{\infty}((0, \infty) \times \mathbb{H})$ in identity (2.3), one has

$$
\left|\int_{0}^{T} \int_{\mathbb{H}} \varphi_{t} \omega^{\varepsilon}(x, t) d x d t\right| \leq C_{\varphi}
$$

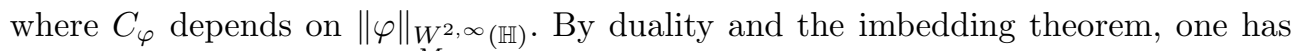
$\omega_{t}$ belongs to $L^{\infty}\left(0, T ; H^{-M}(\mathbb{H})\right)$ for any $T>0$ and $M>3$.

Thanks to the a prior estimate of $\omega^{\epsilon}$ above and utilizing the Aubin-Lions Lemma, there exists a limit $\omega \in L^{\infty}(0, \infty ; B M(\mathbb{H})) \cap C\left([0, T) ; H^{-L}(\mathbb{H})\right)$, for any $T>0$ and some $L<M$, such that, taking a subsequence if necessary, $\omega^{\epsilon} \rightarrow \omega$ weak-* in $L^{\infty}(0, \infty ; B M(\mathbb{H}))$ and $\omega^{\epsilon} \rightarrow \omega$ strongly in $C\left([0, T) ; H^{-L}(\mathbb{H})\right)$. To prove that $\omega$ is a weak solution of (1.1)-(1.3), the only difficulty lies in the nonlinear term

$$
\mathcal{W}_{N L}\left[\omega^{\epsilon}, \varphi\right] \equiv \int_{0}^{T} \int_{\mathbb{H}} \int_{\mathbb{H}} H_{\varphi}(x, y, t) \omega^{\epsilon}(x, t) \omega^{\epsilon}(y, t) d y d x d t .
$$

when we take the limit in the identity (2.3) as $\epsilon \rightarrow 0$. Using Theorem 3.3, together with the boundedness of $H_{\varphi}$ given in Remark 2.2, and the fact that $H_{\varphi}$ is continuous off of the diagonal $x=y$, we can deduce that $\mathcal{W}_{N L}\left[\omega^{\epsilon}, \varphi\right] \rightarrow \mathcal{W}_{N L}[\omega, \varphi]$ as $\epsilon \rightarrow 0$ in a similar arguments given in [1], [17] and [14]. The proof of the theorem is finished.

4. General domains. The above results can be easily generalized to the general domain, which reads as

THEOREM 4.1. Let $\Omega=\left\{\left(x_{1}, x_{2}\right): x_{2} \geq \gamma\left(x_{1}\right)\right\}$, where $\gamma\left(x_{1}\right)=0$ for $\left|x_{1}\right| \geq x_{0}, x_{0}$ is a fixed constant, and $\gamma\left(x_{1}\right)$ is a sufficient smooth and simple curve. Suppose that $\omega_{0} \in B M(\Omega)$ is nonnegative and has a compact support such that $u_{0}=K_{\mathbb{H}}\left[\omega_{0}\right] \in$ $\left(L^{2}(\Omega)\right)^{2}$. Then the result of Theorem 3.4 holds true.

Defining a smooth map, which maps $\gamma$ into a straight line, we can prove Theorem 4.1 in a similar way as Theorem 3.4 (see [14] for details). The main part is to obtain the following estimate on the boundary value of $u$, which is similar to (3.5) in the case of the half plane:

$$
\int_{0}^{T} \int_{\mathcal{K}}|u|^{2} d S d t \leq C
$$

where $\mathcal{K}$ is a compact set of the boundary $\partial \Omega$. 
To prove (4.1), we use the same test function as in (3.5), $\phi=\operatorname{arcctg}\left(x_{1}\right)$. Note that, for this test function, for each compact subset $\mathcal{K}$ of $\partial \Omega$, there exists $c_{0}>0$ such that $\nabla \varphi \cdot n^{\perp} \leq-c_{0}$, i.e., $\nabla^{\perp} \varphi \cdot n \geq c_{0}$ on $\mathcal{K}$. Direct calculations show that $\nabla \varphi=\left(-\left(1+x_{1}^{2}\right)^{-1}, 0\right)$ with $n^{\perp}=(1,0)$ on the straight portion of $\partial \Omega$ and $n^{\perp}=$ $\left(1+\left(\gamma^{\prime}\left(x_{1}\right)\right)^{2}\right)^{-\frac{1}{2}}\left(1, \gamma^{\prime}\left(x_{1}\right)\right)$ on the curved portion of $\partial \Omega$. It is noted that on the curved portion of $\partial \Omega$ the constant $c_{0}$ depends on the bound of $\gamma^{\prime}$. It follows that

$$
\begin{aligned}
\int_{0}^{T} \int_{\mathcal{K}}|u|^{2} d S d t= & \int_{0}^{T} \int_{\mathcal{K}}\left|u \cdot n^{\perp}\right|^{2} d S d t \\
\leq & \frac{1}{c_{0}} \int_{0}^{T} \int_{\mathcal{K}}\left|u \cdot n^{\perp}\right|^{2} \nabla^{\perp} \varphi \cdot n d S d t \\
\leq & \frac{1}{c_{0}}\left(\int_{\Omega} \varphi(x) \omega_{0}(x) d x-\int_{\Omega} \varphi(x) \omega(x, T) d x\right. \\
& \quad+\int_{0}^{T} \int_{\Omega}\left[\left(\left(u_{2}\right)^{2}-\left(u_{1}\right)^{2}\right) \varphi_{x_{1} x_{2}}+u_{1} u_{2}\left(\varphi_{x_{1} x_{1}}-\varphi_{x_{1} x_{2}}\right)\right] d x \\
& \left.\quad+\epsilon \int_{0}^{T} \int_{\Omega} \Delta \varphi \omega d x d t\right) \\
\leq & \frac{1}{c_{0}}\left(2 T\|\varphi\|_{L^{\infty}(\Omega)}\left\|\omega_{0}\right\|_{L^{1}(\Omega)}+T\left\|D^{2} \varphi\right\|_{L^{\infty}(\Omega)}\left\|u_{0}\right\|_{L^{2}(\Omega)}^{2}\right. \\
& \left.\quad+\epsilon T\left\|D^{2} \varphi\right\|_{L^{\infty}(\Omega)}\left\|\omega_{0}\right\|_{L^{1}(\Omega)}\right),
\end{aligned}
$$

where $D^{2} \varphi$ stands for a generic second derivative of $\varphi$.

In the final part of the paper, we point out that by using the Navier-Stokes approximations, we can prove that the existence of weak solutions to (1.1) in an exterior domain with reflection symmetry on the $x_{1}$ - axis under assumptions that the initial vorticty is a vortex-sheets data with reflection symmetry (see [15]). More precisely, let $\Omega=\Omega^{+} \cup \Omega^{-}$, where $\Omega^{+}=\left\{\left(x_{1}, x_{2}\right): x_{2} \geq \sigma\left(x_{1}\right)\right\}$, and $\Omega^{-}=\left\{\left(x_{1}, x_{2}\right)\right.$ : $\left.x_{2} \leq-\sigma\left(x_{1}\right)\right\}$ with $\sigma\left(x_{1}\right)=0$ for $\left|x_{1}\right| \geq x_{0}$, where $x_{0}>0$ is a fixed number, and $\sigma\left(x_{1}\right)$ is a sufficient smooth and simple curve. Then the result of Theorem 3.4 holds true for the exterior problem of the 2D incompressible Euler equations under assumptions that the initial vorticty is a vortex-sheets data with reflection symmetry. In fact, similar to Theorem 2 in [14], the solution $\omega$ is a weak solution of the 2D Euler equations in the upper domain $\Omega^{+}$with initial data $\omega_{0}$ if and only if $\tilde{\omega}$ is a weak solution of the $2 \mathrm{D}$ Euler equations in the full domain $\Omega=\Omega^{+} \cup \Omega^{-}$with initial data $\tilde{\omega}_{0}$, where $\tilde{\omega}$ and $\tilde{\omega}_{0}$ denote the odd extension of $\omega$ and $\tilde{\omega}_{0}$ to the domain $\Omega$ with respect to the variable $x_{1}$, respectively. Thus the vortex sheets problem on $\Omega=\Omega^{+} \cup \Omega^{-}$can be reduced to the vortex sheet problem on $\Omega^{+}$, which has been considered in Theorem 4.1.

\section{REFERENCES}

[1] J. M. Delort, Existence de nappes de tourbillon en dimension deux, J. Amer. Math. Soc., 4 (1991), pp. 553-586.

[2] J. M. DELORT, Une remarque sur le probleme des nappes de tourbillon axisymetriques sur $R^{3}$, J. Funct. Anal., 108 (1992), pp. 274-295.

[3] R. J. DiPerna AND A. J. MAJDA, Concentrations in regularizations for 2-D incompressible flow, Comm. Pure Appl. Math., 40 (1987), pp. 301-345.

[4] L. C. Evans AND S. Müller, Hardy spaces and the two-dimensional Euler equations with nonnegative vorticity, J. Amer. Math. Soc., 7 (1994), pp. 199-219.

[5] D. Iftimie And F. Sueur, Viscous boundary layers for the Navier-Stokes equations with the Navier slip conditions, preprint 2007. 
[6] Q. S. JIU AND D. J. NIU, Vanishing viscous limits for the $2 D$ lake equations with Navier boundary conditions, J. Math. Anal. Appl., 338 (2008), pp. 1070-1080.

[7] Q. S. JIU AND Z. P. XIN, Viscous approximation and decay rate of maximal vorticity function for 3-D axisymmetric Euler equations, Acta Math. Sin. (Engl. Ser.), 20 (2004), pp. 385404.

[8] Q. S. JIU AND Z. P. XIN, On strong convergence to 3-D axisymmetric vortex sheets, J. Differential Equations, 223 (2006), pp. 33-50.

[9] Q. S. JiU AND Z. P. XIN, On strong convergence to $3 D$ steady vortex sheets, J. Differential Equations, 239 (2007), pp. 448-470.

[10] P.-L. Lions, Mathematical topics in fluid mechanics. Vol. 1, volume 3 of Oxford Lecture Series in Mathematics and its Applications, The Clarendon Press Oxford University Press, New York, 1996. Incompressible models, Oxford Science Publications.

[11] J. G. LiU AND Z. P. XIN, Convergence of a Galerkin method for 2-D discontinuous Euler flows, Comm. Pure Appl. Math., 53 (2000), pp. 786-798.

[12] J. G. Liu And Z. P. XIN, Convergence of the point vortex method for 2-D vortex sheets, Math. Comp., 70 (2000), pp. 595-606.

[13] M. C. Lopes Filho, H. J. Nussenzveig Lopes, and G. Planas, On the inviscid limit for two-dimensional incompressible flow with Navier friction condition, SIAM J. Math. Anal., 36 (2005), pp. 1130-1141.

[14] M. C. Lopes Filho, H. J. Nussenzveig Lopes, and Z. P. Xin, Existence of vortex sheets with reflection symmetry in two space dimensions, Arch. Ration. Mech. Anal., 158 (2001), pp. 235-257.

[15] M. C. Lopes Filho, H. J. Nussenzveig Lopes, And Z. P. Xin, Vortex sheets with reflection symmetry in exterior domains, J. Differential Equations, 229 (2006), pp. 154-171.

[16] A. J. MAJDA, Remarks on weak solutions for vortex sheets with a distinguished sign, Indiana Univ. Math. J., 42 (1993), pp. 921-939.

[17] S. Schochet, The weak vorticity formulation of the 2-D Euler equations and concentrationcancellation, Comm. Partial Differential Equations, 20 (1995), pp. 1077-1104.

[18] R. Temam, Navier-Stokes equations. Theory and numerical analysis. North-Holland Publishing Co., Amsterdam, 1977. Studies in Mathematics and its Applications, Vol. 2.

[19] I. Vecchi And S. J. Wu, On $L^{1}$-vorticity for 2-D incompressible flow, Manuscripta Math., 78 (1993), pp. 403-412.

[20] Y. L. XIAO AND Z. P. XIN, On the vanishing viscosity limit for the 3D Navier-Stokes equations with a slip boundary condition, Comm. Pure Appl. Math., 60 (2007), pp. 1027-1055. 
D. NIU, Q. JIU AND Z. XIN 